\title{
Modelling Risk to US Military Populations from Stopping Blanket Mandatory Polio Vaccination
}

\author{
Colleen Burgess, ${ }^{1,2}$ Andrew Burgess, ${ }^{2}$ and Kellie McMullen ${ }^{3}$ \\ ${ }^{1}$ Ramboll Environ, Inc., Amherst, MA, USA \\ ${ }^{2}$ MathEcology, LLC, Phoenix, AZ, USA \\ ${ }^{3}$ Naval Health Research Center, San Diego, CA, USA
}

Correspondence should be addressed to Colleen Burgess; cburgess@ramboll.com and Kellie McMullen; kellie.l.mcmullen2.mil@mail.mil

Received 27 April 2017; Accepted 8 June 2017; Published 14 September 2017

Academic Editor: Delfim F. M. Torres

Copyright (C) 2017 Colleen Burgess et al. This is an open access article distributed under the Creative Commons Attribution License, which permits unrestricted use, distribution, and reproduction in any medium, provided the original work is properly cited.

\begin{abstract}
Objectives. Transmission of polio poses a threat to military forces when deploying to regions where such viruses are endemic. USborn soldiers generally enter service with immunity resulting from childhood immunization against polio; moreover, new recruits are routinely vaccinated with inactivated poliovirus vaccine (IPV), supplemented based upon deployment circumstances. Given residual protection from childhood vaccination, risk-based vaccination may sufficiently protect troops from polio transmission. Methods. This analysis employed a mathematical system for polio transmission within military populations interacting with locals in a polio-endemic region to evaluate changes in vaccination policy. Results. Removal of blanket immunization had no effect on simulated polio incidence among deployed military populations when risk-based immunization was employed; however, when these individuals reintegrated with their base populations, risk of transmission to nondeployed personnel increased by $19 \%$. In the absence of both blanket- and risk-based immunization, transmission to nondeployed populations increased by $25 \%$. The overall number of new infections among nondeployed populations was negligible for both scenarios due to high childhood immunization rates, partial protection against transmission conferred by IPV, and low global disease incidence levels. Conclusion. Risk-based immunization driven by deployment to polio-endemic regions is sufficient to prevent transmission among both deployed and nondeployed US military populations.
\end{abstract}

\section{Introduction}

Polio is a viral disease that invades the nervous system and can cause paralysis in a matter of hours, though most poliovirus infections are asymptomatic [1]. There are 3 wild poliovirus (WPV) types, two of which have not been detected globally since 2012 [2]. Polio infection in immunocompetent individuals leads to immunity, although immunity induced by one serotype does not protect against the other two [3]. There are 2 vaccines against polio: inactivated poliovirus vaccine (IPV) and oral poliovirus vaccine (OPV). Successful vaccination with either formulation provides at least partial protection from infection and full disease immunity in approximately 7 days [4].

After IPV immunization, antibodies are produced in the blood in response to the inactivated virus, protecting the individual from disease; however, viral replication in the gut is still possible, with the potential for asymptomatic transmission to the community [5]. IPV-induced antibodies decrease over time, and some adults vaccinated as children may lack a detectable antibody.

In contrast, OPV induces mucosal immunity in the gastrointestinal tract, which is important for community protection [6], and provides long-term immunity against both disease and transmission. Yet, "the oral vaccine often fails in developing countries and in rare cases the vaccine virus itself leads to paralysis" [7]. OPV can also lead to the circulation of a vaccine-induced virus, shed for $1-3$ weeks following primary vaccination and easily transmitted within and outside the household. While this shedding is widely accepted as inducing protection or boosting immunity in contacts [3], 
it can also contribute to the spread of circulating vaccinederived poliovirus (cVDPV) [6], particularly in settings with weak routine immunization coverage in otherwise polio-free countries [8].

Due to these risks, most developed countries have switched from OPV to IPV [9]. However, for developing countries where polio is endemic or the risk of importation is high, the benefits of OPV outweigh the risks, and, for now, OPV remains the vaccine of choice [3]. The ultimate goal is to eliminate OPV vaccination and switch to IPVonly immunization, thus eliminating the risk of cVDPV altogether. As of November 2016, 173 countries now include IPV in their routine immunizations; the remaining World Health Organization (WHO) member countries are on schedule to introduce IPV by the end of 2016 [10].

Today, WPV remains endemic in 3 countries-Pakistan, Afghanistan, and Nigeria [11]. While WPVs circulate in these areas, "the rest of the world must continue to keep polio vaccination levels very high, due to the risk of outbreaks among susceptible people in polio-free countries" [12]. Response to polio circulation in developing areas such as these generally involves the use of OPV, and "each response round comes with a substantial and uncertain amount of secondary OPV infection" [4], which are of particular risk to deployed US soldiers with incomplete or waning protection against poliomyelitis transmission.

In the United States, children currently receive 3 routine doses of IPV at 2 months, 4 months, and 6-18 months, and a booster at 4-6 years [13]. As a result, US-born soldiers generally enter military service with a high level of immunity to disease, though this may wane over time. The military actively vaccinates all recruits against a number of diseases including polio; "these vaccines are further supplemented, based on occupational and deployment circumstances of the recruits" [14]. However, in countries with insufficient vaccination and/or active viral circulation, contact with local populations puts US warfighters at risk for transmission of both WPV and cVDPV. This is particularly the case since

[although] militaries primarily engage in traditional major combat operations, they are increasingly involved in humanitarian assistance missions. Such missions permit extensive interaction with the local populace and environment, greatly increasing the chance of acquiring locally endemic infectious diseases and necessitating the management of diseases in the local populace that are not traditionally seen in military personnel [15].

Thus, while blanket vaccination of all soldiers may be epidemiologically redundant and cause unnecessary expenditure of resources, risk-based vaccination driven by travel to polio-endemic areas can be appropriate. However, evaluating multiple vaccination strategies once deployment is underway may result in higher-than-necessary disease incidence and cost in an effort to control transmission. In lieu of this, predictive modelling of polio transmission allows for the exploration of vaccination strategies through simulation of multiple scenarios and their outcomes prior to putting troops and mission objectives at risk.

In recent years, published mathematical models for polio transmission have focused primarily on the role of OPV in attaining eradication of the disease. Several mathematical models have also explored vaccine-derived polioviruses [1618], and additional analyses have addressed the impact of asymptomatic infection [19-22]. Kalkowska et al. [20, 21] explored the possibility of silent transmission of WPV in populations with high IPV coverage, emphasizing that "IPV-based protection alone might not provide sufficient population immunity to prevent poliovirus transmission after an importation" [20] and acknowledging the need to "consider the role of previously-vaccinated or infected individuals (i.e., partially infectible individuals) who remain immune to paralytic disease, but not to reinfection, and their potential participation in silent transmission of the virus" [21]. Most recently, Koopman et al. [22] modelled the interaction between waning immunity and the duration of silent circulation of polio and found that expanding beyond childhood immunization to vaccinate a portion of the adult population could have a significant impact on asymptomatic transmission.

Each of these modelling studies focuses on issues among multigenerational populations with varying levels of immunization coverage and background immunity; none have addressed the unique circumstances affecting polio transmission in highly mobile military populations, which experience conditions that directly affect the spread of disease, both beneficially and detrimentally. US soldiers are vaccinated against polio at recruitment and again prior to deployment to atrisk areas; however, immunization with IPV may leave troops at risk of VDPV or subject to asymptomatic transmission. Quantifying this risk is crucial to evaluating the elimination of mandatory blanket polio vaccination and switching to a solely risk-based vaccination policy.

Mathematical models can be immensely useful in examining the impact of vaccines on disease transmission and are frequently used to inform response policy. For deployed military populations, these models can also evaluate the relative change in transmission risk associated with multiple vaccination scenarios by employing data on specific demographics, epidemiology, and the effects of timing of response. For this analysis, we modified an existing military-specific model system to accommodate polio transmission and vaccination to maximize the achievement of deployed mission objectives, while minimizing the possibility of transmission to troops both abroad and at home.

\section{Methods}

2.1. Population Structure. The military population structure for this study was built upon previous analyses [23]. The simulated deployed population consisted of 4 subpopulations defined by interaction with the local population, ranging from negligible to high levels of daily contact. Deployed soldiers were assumed to be posted to a long-standing base 


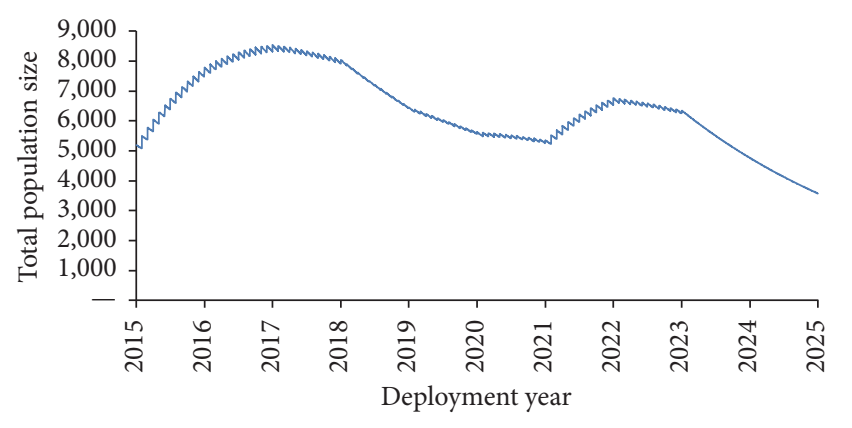

Figure 1: Change in deployed population size over the 10-year duration of the deployment action [23].

within the host country, with well-established water purification and food safety systems to minimize environmental disease transmission. Social mixing was presumed to be mainly within-unit and homogeneous, with between-unit mixing occurring at lower levels.

The 10-year simulated deployment period began in 2015, with individual soldiers rotating annually. In- and outbound rotation rates $\left(b_{\mathrm{IN}}\right.$ and $\left.b_{\mathrm{OUT}}\right)$ varied over the 10 -year period to allow for force increase and decrease (see Figure 1) and a daily casualty rate $(\mu)$ accounted for removal of individuals for reasons other than rotation or polio-related disease. See Burgess et al. [23] for additional population details.

The local population was modelled without structure and based on the demographic characteristics of Afghanistan as estimated by the United Nations Population Division [24]. Mixing among locals was homogeneous within the simulated geographic region. Local birth and nonpolio death rates varied annually [24], and a polio-specific death rate $\left(\mu_{\text {POLIO }}\right)$ was applied to infected individuals. OPV immunization of locals was set to historic reported coverage rates $(\rho)$ up to 2013 (the most recent year for which data was available at the time of model development) [25] and held constant at the 2013 rate for the remainder of the simulation period.

2.2. Transmission Model. Polio transmission was modelled as a modified compartmental Susceptible-Exposed-InfectedRemoved model (1) (see Figure 2 and Tables 1 and 2) employing structured ordinary differential equations. Transmission of polio occurred via direct contact between susceptible and infected individuals, with random case importation boosting infection levels stochastically throughout the simulation.

$$
\begin{aligned}
\frac{d S_{i}}{d t}= & b_{\mathrm{IN} i}\left(1-\text { protect }_{i}\right) N_{i} \\
& -\left(\rho_{i}+\beta_{i}+\mu_{i}+b_{\mathrm{OUT} i}\right) S_{i}, \\
\frac{d S_{P i}}{d t}= & b_{\mathrm{IN} i} \operatorname{protect}_{i} N_{i}+\Omega R_{i}-\left(\pi_{s} \beta_{i}+\mu_{i}+b_{\mathrm{OUT} i}\right) S_{P i}, \\
\frac{d E_{i}}{d t}= & \beta_{i} S_{i}-\left(\iota+\mu_{i}+b_{\mathrm{OUT} i}\right) E_{i},
\end{aligned}
$$

\begin{tabular}{|c|c|}
\hline Variable & Definition \\
\hline$S_{i}$ & $\begin{array}{l}\text { Number of unprotected susceptible individuals in } \\
\text { subpopulation } i\end{array}$ \\
\hline$S_{P i}$ & $\begin{array}{l}\text { Number of partially protected susceptible individuals } \\
\text { in subpopulation } i\end{array}$ \\
\hline$E_{i}$ & $\begin{array}{l}\text { Number of unprotected exposed individuals in } \\
\text { subpopulation } i\end{array}$ \\
\hline$E_{P i}$ & $\begin{array}{c}\text { Number of partially protected exposed individuals in } \\
\text { subpopulation } i\end{array}$ \\
\hline$I_{i}$ & $\begin{array}{l}\text { Number of unprotected infected individuals in } \\
\text { subpopulation } i\end{array}$ \\
\hline$I_{P i}$ & $\begin{array}{c}\text { Number of partially protected infected individuals in } \\
\text { subpopulation } i\end{array}$ \\
\hline$R_{i}$ & $\begin{array}{l}\text { Number of recovered or removed individuals in } \\
\text { subpopulation } i\end{array}$ \\
\hline$N_{i}$ & Total population size in subpopulation $i$ \\
\hline
\end{tabular}

TABLE 1: Variable definitions for polio transmission model.

$$
\begin{aligned}
\frac{d E_{P i}}{d t} & =\pi_{s} \beta_{i} S_{P i}-\left(\iota_{p}+\mu_{i}+b_{\mathrm{OUT} i}\right) E_{P i}, \\
\frac{d I_{i}}{d t} & =\iota E_{i}-\left(\gamma+\mu_{\mathrm{POLIO}}+\mu_{i}+b_{\mathrm{OUT} i}\right) I_{i}, \\
\frac{d I_{P i}}{d t} & =\iota_{p} E_{P i}-\left(\gamma_{p}+\mu_{i}+b_{\mathrm{OUT} i}\right) I_{P i}, \\
\frac{d R_{i}}{d t} & =\rho_{i} S_{i}+\gamma I_{i}+\gamma_{p} I_{P i}-\left(\Omega+\mu_{i}+b_{\mathrm{OUT} i}\right) R_{i}, \\
\frac{d N_{i}}{d t} & =\left(b_{\mathrm{IN} i}-b_{\mathrm{OUT} i}-\mu_{i}\right) N_{i}-\mu_{\mathrm{POLIO}} I_{i} .
\end{aligned}
$$

To establish endemicity, polio transmission among locals was simulated for a burn-in period of 35 years prior to the arrival of the deployed military population in 2015. Simulated, combined symptomatic and asymptomatic incidence during this burn-in phase was validated against reported paralytic polio cases as recorded by the WHO [34], adjusted to account for the widely accepted $10 \%$ proportion of cases that are symptomatic [28]. Validation was performed qualitatively and visually, comparing adjusted historical data to the output of multiple stochastic model iterations under baseline parameter assumptions. Simulated local cases fit well with historical data for 1980-2014 (data missing for 1992-1994 and 1996), in terms of both magnitude and frequency of peaks (Figure 3).

Individuals entered population either via birth (locals) with full susceptibility $(S)$ or via inward rotation (military) with partial susceptibility $\left(S_{p}\right)$ defined by childhood immunization, blanket immunization upon recruitment, and/or booster immunization prior to deployment (see Scenario Construction). Following an incubation period of 3-4 days ( $\iota$ or $\iota_{p}$, depending on immune status), exposed individuals $\left(E, E_{p}\right)$ progressed to infection lasting 27 days $(Y)$ (range: 27-28 days) for unprotected individuals $(I)$ or 9 days $\left(\Upsilon_{p}\right)$ (range: 9-25 days) for partially protected individuals $\left(I_{p}^{p}\right)$, who were $20 \%\left(\pi_{i}\right)$ (range: $20-90 \%$ ) as infectious as fully 


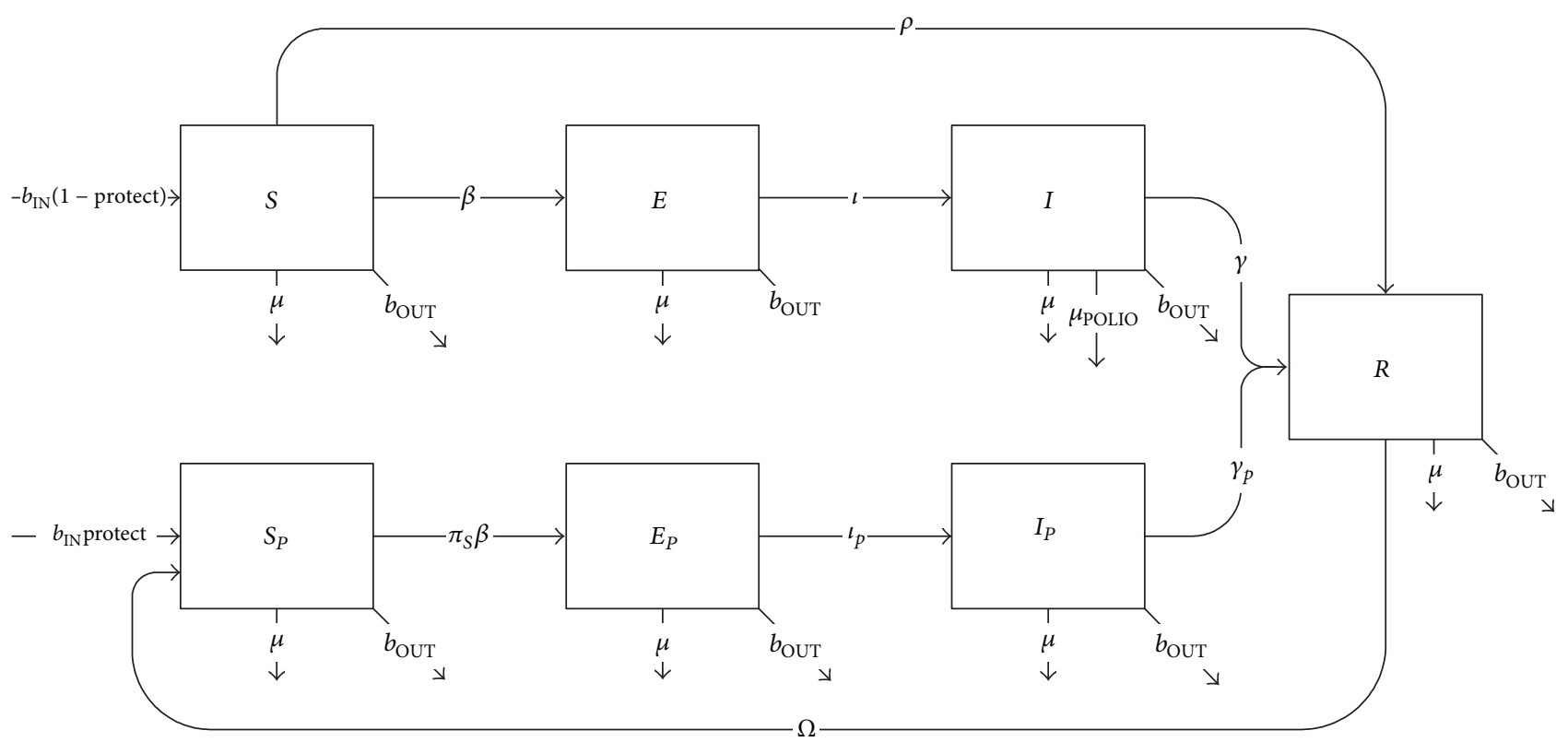

FIGURE 2: Schematic diagram of polio transmission with both OPV $(\rho)$ and IPV $\left(S_{p}\right)$ immunization and waning immunity.

TABLE 2: Parameter definitions for polio transmission model for military (MIL) and local (LOC) populations.

\begin{tabular}{|c|c|c|c|}
\hline Parameter & Definition & Value (range) & Source \\
\hline$b_{\mathrm{IN} i}$ & $\begin{array}{l}\text { Daily inbound rotation rate (MIL)/birth rate (LOC) for } \\
\text { subpopulation } i\end{array}$ & [Array] & \\
\hline$b_{\mathrm{OUT} i}$ & $\begin{array}{l}\text { Daily outbound rotation rate (MIL)/background death rate (LOC) for } \\
\text { subpopulation } i\end{array}$ & [Array] & \\
\hline$\mu_{i}$ & Daily background casualty rate for subpopulation $i$ (MIL) & [Array] & \\
\hline protect $_{i}$ & $\begin{array}{l}\text { Daily proportion of inbound population with preexisting partial } \\
\text { protection for subpopulation } i \text { (MIL) }\end{array}$ & [Array] & \\
\hline$\rho_{i}$ & Daily (OPV) vaccination rate for subpopulation $i$ (LOC) & [Array] & \\
\hline$\beta_{i}$ & Effective polio transmission rate for subpopulation $i$ & [Function] & \\
\hline$\pi_{s}$ & Relative susceptibility of $S_{p}$ individuals & $0.2(0.2-0.9)$ & {$[26]$} \\
\hline $1 / \iota$ & Duration of latent period for unprotected individuals & $3 d(3-4 d)$ & {$[26,27]$} \\
\hline $1 / \iota_{p}$ & Duration of latent period for partially protected individuals & $4 \mathrm{~d}(3-4 \mathrm{~d})$ & {$[26,27]$} \\
\hline $1 / \gamma$ & Duration of infectious period for unprotected individuals & $27 \mathrm{~d}(27-28 \mathrm{~d})$ & {$[26]$} \\
\hline $1 / \gamma_{p}$ & Duration of infectious period for partially protected individuals & $9 d(9-25 d)$ & {$[26]$} \\
\hline$\mu_{\text {POLIO }}$ & Polio mortality rate & $0.22(0.02-0.30)$ & {$[28]$} \\
\hline$\Omega$ & 1/duration of OPV or disease-induced immunity & $1 /(365 * 20)$ & (See text) \\
\hline seas & Seasonal variation in polio transmission & [Function] & \\
\hline$\sigma$ & Proportional change in polio transmission due to seasonality & 0.15 & {$[21]$} \\
\hline$\chi$ & Polio attack rate & $\begin{array}{c}20 / 100,000 \\
(0.1 / 1,000,000-6.8 / 100,000)\end{array}$ & {$[29-32]$} \\
\hline$C_{i j}$ & Daily contact rate between subpopulations $i$ and $j$ & [Array] & {$[23]$} \\
\hline$\pi_{i}$ & Relative infectiousness of $I_{p}$ individuals & $0.2(0.2-0.9)$ & {$[26,33]$} \\
\hline symp & Proportion of unprotected polio cases that are symptomatic & 0.1 & {$[28]$} \\
\hline importrisk & Probability of polio case importation from outside population & 0.001 & (See text) \\
\hline importampl & Amplitude of polio case importation from outside population & $2 / 100,000$ & (See text) \\
\hline
\end{tabular}




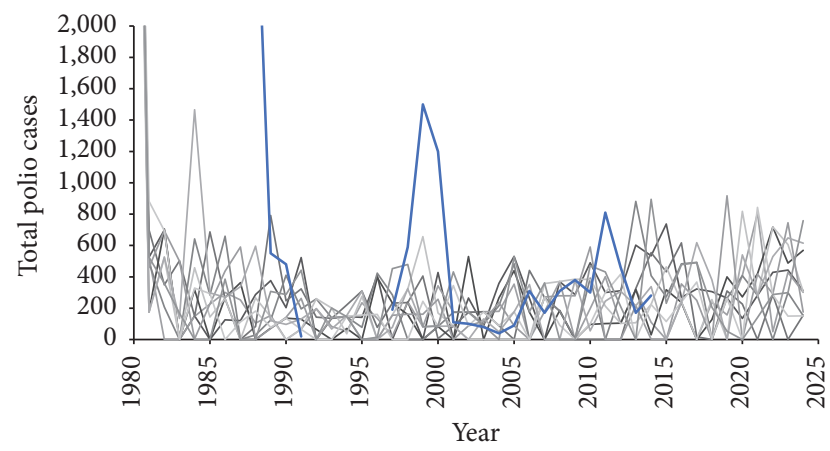

FIGURE 3: Qualitative comparison between simulated (gray lines) and historical (blue line) polio cases (symptomatic + asymptomatic) for the local population over a sample of 10 random simulations.

unprotected infected persons. Recovered individuals $(R)$ possessed immunity to both transmission and disease for a period of 20 years $(1 / \Omega)$ (see discussion of uncertainty), after which only immunity to disease was retained and individuals entered (or reentered) the partially susceptible $\left(S_{p}\right)$ class.

Ten percent (symp) of cases in nonimmune, infected individuals presented with symptoms, with the remainder being subclinical; all infections in partially protected individuals were assumed to be asymptomatic. Polio-related mortality affected only symptomatically infected individuals.

Polio transmission was driven by an attack rate $(\chi)$ of 20 cases per 100,000 population (range: $0.1 / 1,000,000-20 / 100,000$ ) with seasonal forcing (seas), allowing for both high- and low-transmission periods within the year. Transmission was further impacted by stochastic case importation with $0.1 \%$ probability (importrisk) and amplitude of $1 / 10$ th the attack rate (importampl) (see discussion of uncertainty):

$$
\begin{aligned}
\text { seas }= & 1+\sigma\left(\cos \left(\frac{2 \pi t}{365}\right)\right), \\
\beta_{i}= & \left(\text { seas } * \chi * \sum C_{i j}\left(\frac{I_{j}+\pi_{i} I_{P j}}{N_{j}}\right)\right) \\
& + \text { import, } \\
\text { import }= & \begin{cases}\text { importampl } & \text { if rand }<\text { importrisk } \\
0 & \text { if rand } \geq \text { importrisk, }\end{cases}
\end{aligned}
$$

where rand $\sim U(0,1)$.

2.3. Sensitivity and Uncertainty Analyses. Sensitivity and uncertainty analyses were performed on 21 model parameters, with outcomes measured in terms of total symptomatic and asymptomatic polio cases and mean annual incidence among military and local populations.

Total military cases and annual incidence showed direct sensitivity to the relative susceptibility $\left(\pi_{s}\right)$ of partially protected individuals $\left(S_{p}\right)$. Since the significant majority of the military population falls into the $S_{p}$ category, as a result of IPV immunization, it is logical that increased relative susceptibility in these individuals results in more cases overall. In contrast, transmission among locals was dramatically less sensitive to $\pi_{s}$, since the only $S_{p}$ individuals within the local population included those who were previously infected and recovered and for whom enough time had passed that preexisting immunity from recovery waned.

Local annual disease incidence was significantly sensitive to the polio death rate $\left(\mu_{\text {pOLIO }}\right)$; higher mortality results in fewer polio cases, as a result of an overall decrease in individuals participating in transmission. As IPV immunization is assumed to confer at least partial protection, the bulk of polio cases within deployed troops are asymptomatic and, thus, not affected by disease-related death rates.

The duration of the infectious period $\left(1 / \Upsilon_{p}\right)$ for partially protected, infected individuals $\left(I_{p}\right)$ significantly affected total military cases and annual incidence, reflecting that a shorter infectious period among $I_{p}$ resulted in lower transmission and, therefore, fewer subsequent polio cases. The impact on local populations was dramatically lower due to the smaller fraction of locals falling within the $I_{p}$ category.

Local incidence was inversely affected by changes in the duration of OPV- or disease-induced immunity $(1 / \Omega)$, that is, longer-lasting immunity resulted in fewer local cases overall. There was no significant impact on military polio incidence; however, since these individuals were not immunized with OPV, the overall deployment period was not long enough to allow for waning of postrecovery immunity to occur.

The level of residual protection resulting from childhood IPV immunization (childhood) had a moderate impact on polio transmission within military populations in simulations within which no other polio vaccination was administered. In the presence of either blanket or booster vaccination (or both), variation in childhood residual protection had no impact on military polio transmission, since the more recent adult immunization overrode any effects due to vaccination occurring early in life.

Uncertainty in case importation (importrisk and importampl) had a dramatic impact on polio incidence in both military and local populations, overriding even variation in the polio attack rate $(\chi)$. This mirrors on-the-ground experience with polio eradication efforts. In the absence of new cases entering into an area, adequate routine immunization can halt the local transmission of polio; however, small levels of case importation can spark outbreaks even in immunized regions.

2.4. Scenario Construction. To evaluate the hypothesis that residual immunity from childhood vaccination, combined with risk-based deployment vaccination, is sufficient to protect troops from polio transmission, 3 military IPV scenarios were tested:

(1) Blanket immunization at recruitment + booster immunization at deployment + residual childhood protection (Scenario 1, baseline).

(2) (Blanket immunization terminated in 2015) + booster immunization at deployment + residual childhood protection (Scenario 2). 
(3) (Blanket immunization terminated in 2015) + residual childhood protection only (Scenario 3).

As of 2013, the US childhood IPV vaccination coverage rate was approximately $93 \%$ [25]; that is, 93\% of children aged 1-4 years have received 3 routine doses of IPV. The Centers for Disease Control and Prevention's Pink Book [28] indicates that IPV efficacy after 3 doses is approximately $99 \%$ (efficacy), and immunity against disease is "probably lifelong"; while there is some debate regarding waning protection, few studies have been performed to evaluate this. To determine residual adult protection resulting from childhood immunization, we developed a waning curve based on data provided by Lapinleimu and Stenvik [35], which describe the change in detectable polio antibodies over time in individuals in Finland where IPV is the only implemented vaccination. From this data, we extrapolated a relationship between detectable antibodies and years since the most recent IPV booster, defined as a function of age. Based on the 2012 Demographics Profile of the Military Community [36], the average age of the active duty force is 28.7 years; when combined with the childhood IPV coverage rate and applied to the waning immunity curve, on average $92 \%$ of soldiers still possess detectable antibodies resulting from childhood IPV immunization (childhood).

The childhood IPV coverage rate accounts for both philosophical and medical exemptions to vaccination, including impaired immune status, allergies to vaccine components, or history of vaccine-associated adverse events. Within the 20122013 school year, medical exemptions for childhood vaccinations (not specific to polio) ranged from 0.1 to $1.6 \%$ (median $0.3 \%$ ), and nonmedical exemptions ranged from 0.2 to $6.4 \%$ (median 1.5\%) [37]. While immunization exemptions are allowed for military service members, "noncompliance with immunization requirements may adversely impact deployability, assignment, or international travel," and "[nonmedical] exemptions may be revoked, in accordance with servicespecific policies and procedures, if the individual and/or unit are at imminent risk of exposure to a disease for which an immunization is available" [38].

For this analysis, we assumed that military exemption rates sit at the low-end of the range for childhood vaccination exemptions, yielding an overall military IPV exemption rate (exemption) of $0.3 \%$ (range $0.3-8.0 \%$, median $1.8 \%$ ), with the inclusion of a $75 \%$ proportional reduction (dfact) in exemption rate (range $0-100 \%$ ) for deploying personnel.

Military IPV effective coverage rates for blanket and booster immunization were calculated as follows:

$$
\begin{aligned}
\text { blanket } & =(1-\text { exemption }) * \text { efficacy, } \\
\text { boost } & =(1-(\text { dfact } * \text { exemption })) * \text { efficacy. }
\end{aligned}
$$

For Scenarios 2 and 3, blanket immunization upon recruitment was halted in 2015; those individuals recruited prior to 2015 would have relatively recent immunity, while those recruited in 2015 or later would have immunity resulting only from deployment booster immunization (Scenario 2) or residual immunity from childhood immunization (Scenario 3). Thus, for these two scenarios, at any point in time from
2015 through the end of the simulation, there would always be a blend of blanket- and nonblanket-immunized individuals in the deployed population.

As of 2006 [39], accession rates within branches of the US military ranged from 13\% for the Air Force to $19 \%$ for the Army (accession). For Scenarios 2 and 3, the proportion of service members protected by blanket immunization administered prior to $2015(B(t))$ was defined as a recursive function diluted by the accession rate:

$$
B(t)=\left(1-\frac{\text { accession }}{365}\right) * B(t-1) .
$$

The net protection level of the military population from prior blanket immunization combined with residual immunity from childhood immunization was, therefore, given as a maximum function, which decayed to no lower than the childhood protection level:

$$
\begin{aligned}
& N(t)=\max ((B(t) * \text { blanket }) \\
& \quad+[(1-B(t)) * \text { childhood }], \text { childhood })
\end{aligned}
$$

This resulted in a drop in overall protection from $98.7 \%$ to 93.0\% over the 10 -year deployment period.

Scenario-specific parameters are provided in Table 3, and final scenario definitions for deployed and nondeployed military personnel are given in Table 4 .

Each scenario was run for 1500 simulations to account for stochasticity in polio case importation. Model outputs were measured as total deployed symptomatic and asymptomatic polio cases and average annual incidence (included both symptomatic and asymptomatic cases) for deployed military and local populations.

\section{Results}

For all scenarios, local disease dynamics remained fairly consistent across all simulations, tracking with historical cases prior to 2015, then sustaining low endemicity driven by case importation to the end of the simulation period (Figures 4(a)-4(c)).

Similarly, there was insignificant change in polio dynamics among deployed military populations between Scenarios 1 and 2, with a slight increase in infections under Scenario 3 (Figures 5(a)-5(c)).

Stochasticity associated with case importation caused variation between simulation results for total cases and average annual polio incidence for all 3 scenarios (Figures 6(a)-6(d)). This variation was relatively small; however, and data points were generally well-clustered around mean values.

Dropping blanket immunization but maintaining predeployment booster immunization had negligible effect on simulated deployed military cases and incidence. Dropping both blanket and predeployment immunizations yielded a $5 \%$ increase in polio cases and annual incidence among deployed populations over the baseline scenario of blanket immunization (Table 5). Local annual incidence was not significantly affected by changes in military immunization 
TABLE 3: Parameter definitions for scenario calculations.

\begin{tabular}{|c|c|c|c|}
\hline Parameter & Definition & Value (range) & Source \\
\hline efficacy & IPV vaccine efficacy (MIL) & $99 \%(50-100 \%)$ & {$[28]$} \\
\hline childhood & Residual protection level from childhood IPV vaccination & $0.92(0.0-1.0)$ & $\begin{array}{l}\text { Calculated from } \\
\quad[25,35]\end{array}$ \\
\hline exemption & $\begin{array}{l}\text { Overall military vaccination exemption rate (medical + } \\
\text { administrative) }\end{array}$ & $0.003(0.003-0.08)$ & Calculated from [37] \\
\hline dfact & $\begin{array}{l}\text { Proportional reduction in exemption rate for deployed personnel } \\
\text { (versus nondeployed) }\end{array}$ & $0.75(0.0-1.0)$ & (Estimated) \\
\hline blanket & $\begin{array}{c}\text { IPV blanket vaccine coverage (when implemented) for all military } \\
\text { personnel upon accession }\end{array}$ & (Function) & \\
\hline boost & $\begin{array}{c}\text { IPV boost vaccine coverage (when implemented) for deploying } \\
\text { personnel }\end{array}$ & (Function) & \\
\hline accession & Military accession rate & $0.19(0.13-0.19)$ & [39] \\
\hline$B$ & $\begin{array}{c}\text { Proportion of military population covered by blanket vaccination } \\
\text { prior to } 2015\end{array}$ & (Function) & \\
\hline$N$ & $\begin{array}{l}\text { Overall protection of military population from blanket vaccination } \\
\text { prior to } 2015 \text { and residual childhood immunity }\end{array}$ & (Function) & \\
\hline
\end{tabular}

TABLE 4: Scenario definitions for deployed and nondeployed military personnel.

\begin{tabular}{lcc}
\hline & \multicolumn{2}{c}{ Protection levels (protect) } \\
& Deployed personnel & Nondeployed personnel \\
\hline $\begin{array}{l}\text { Scenario 1 } \\
\text { (baseline) }\end{array}$ & blanket & blanket \\
$\begin{array}{l}\text { Scenario 2 } \\
\text { (booster) }\end{array}$ & boost & $N(t)$ \\
$\begin{array}{l}\text { Scenario 3 } \\
\text { (childhood) }\end{array}$ & $N(t)$ & $N(t)$ \\
\hline
\end{tabular}

strategies, with any variation resulting only from model stochasticity, which indicated that transmission from military to local populations was not an important issue at these levels of military protection.

The total number of symptomatic and asymptomatic polio cases in the deployed military population remained less than one for all 3 immunization scenarios, though fractional cases were still utilized in the calculation of incidence rates. Though frequently undetectable in the field, asymptomatic cases were included in the case-count and incidence calculations to provide a measure of the potential for silent transmission.

For nondeployed personnel, dropping blanket immunization resulted in a decrease in polio disease protection from $99 \%$ to $93 \%$. Since IPV vaccination confers full protection from disease but only partial (model assumption: 20\%) protection from transmission, this yielded an increase in overall susceptibility to transmission among the nondeployed population from $21 \%$ to $26 \%$.

Combining nondeployed susceptibility levels with the average annual polio incidence among deployed populations for each scenario allowed for estimation of the risk of new polio infections within nondeployed personnel due to mixing with infected soldiers returning from deployment. For the blanket immunization scenario (Scenario 1), the risk of new polio infections resulting from reintegrating infected soldiers was predicted to be $0.000504 / 1,000,000$. For Scenario 2-where blanket immunization was terminated but predeployment booster was still employed-the simulated risk of new polio infections increased from $0.000504 / 1,000,000$ to $0.000624 / 1,000,000$ after 10 years without blanket vaccination. For Scenario 3-where both blanket and booster immunizations were terminated-simulated risk of new polio infections among nondeployed personnel increased from $0.000546 / 1,000,000$ to $0.000676 / 1,000,000$ (Table 6) over the same 10 -year period.

\section{Discussion and Conclusions}

Mathematical models can help guide preventive medicine policy, resulting in healthier and protected populations. This analysis employed a mathematical model for the transmission of polio within deployed military populations interacting with local populations in an endemic setting. Results from model simulations described the potential benefits of protecting these troops via routine blanket immunization, predeployment booster immunization, and residual protection resulting from childhood vaccination.

In the absence of blanket immunization on recruitment, immunity to polio disease among nondeployed personnel defaults gradually to residual protection resulting from childhood immunization, and the percentage of this population susceptible to transmission increases. Although the removal of simulated blanket immunization had no noticeable effect on polio incidence among deployed personnel subject to predeployment booster immunization, the risk of transmission to nondeployed personnel mixing with deployed soldiers reintegrating with base populations increased by $19 \%$. In the absence of predeployment booster immunization, risk of transmission to nondeployed populations increased by $25 \%$ over the baseline scenario.

Though the increased percentage in transmission resulting from dropping blanket immunization was nonzero, the 


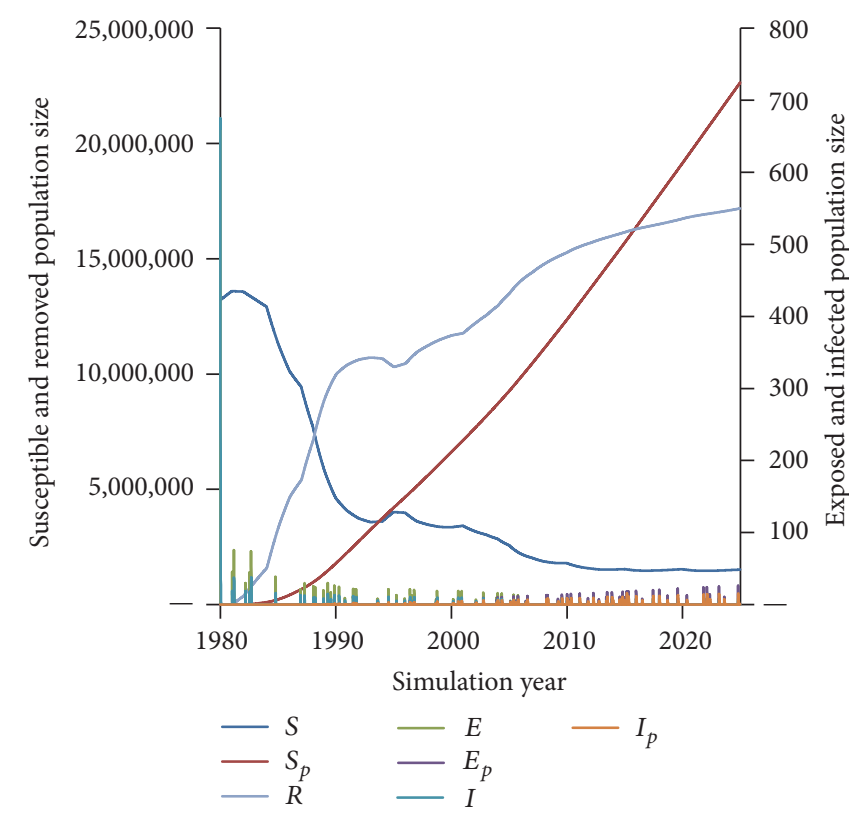

(a)

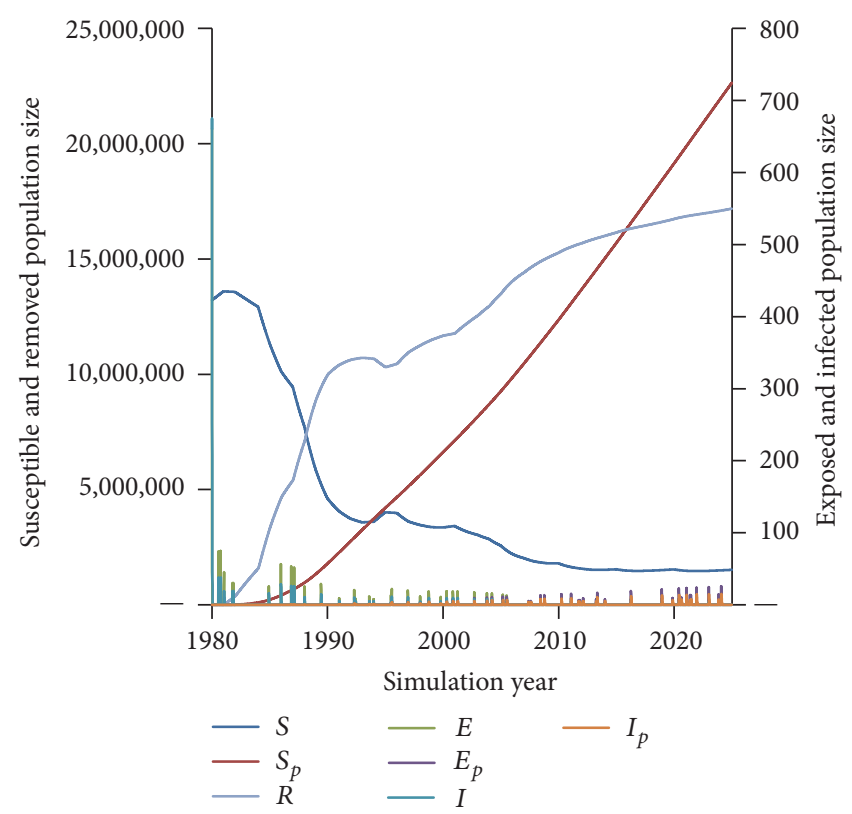

(b)

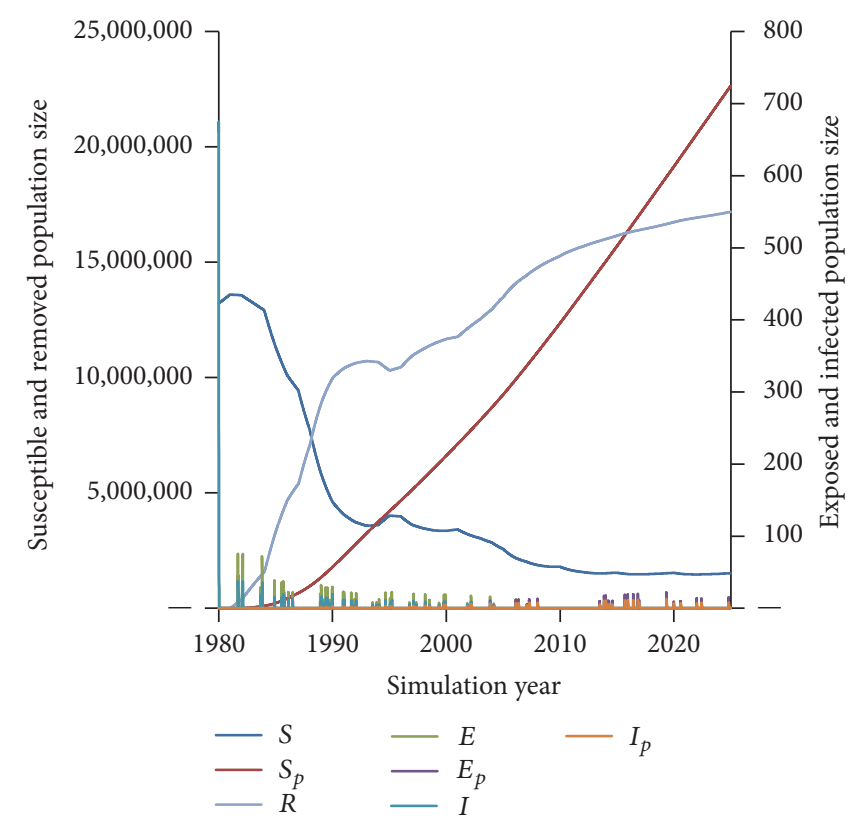

(c)

Figure 4: Polio disease dynamics among local populations under military immunization Scenarios (a) 1 (blanket + booster + residual childhood), (b) 2 (booster + residual childhood), and (c) 3 (residual childhood).

TABLE 5: Median total polio cases and average annual incidence with percentage of change over baseline for deployed military and local populations under 3 military immunization scenarios.

\begin{tabular}{lccrr}
\hline \multirow{2}{*}{ Scenario } & \multicolumn{2}{c}{ Total cases (military) } & \multicolumn{2}{c}{ Average annual incidence } \\
& Symptomatic & Asymptomatic & $0.012 / 1000$ & Local \\
\hline 1 & 0.076 & 0.687 & $0.022 / 1000$ \\
2 & $0.076(+0 \%)$ & $0.687(+0 \%)$ & $0.012 / 1000(+0.2 \%)$ & $0.022 / 1000(+0.8 \%)$ \\
3 & $0.080(+5 \%)$ & $0.721(+5 \%)$ & $0.013 / 1000(+5.4 \%)$ & $0.022 / 1000(+0.1 \%)$ \\
\hline
\end{tabular}



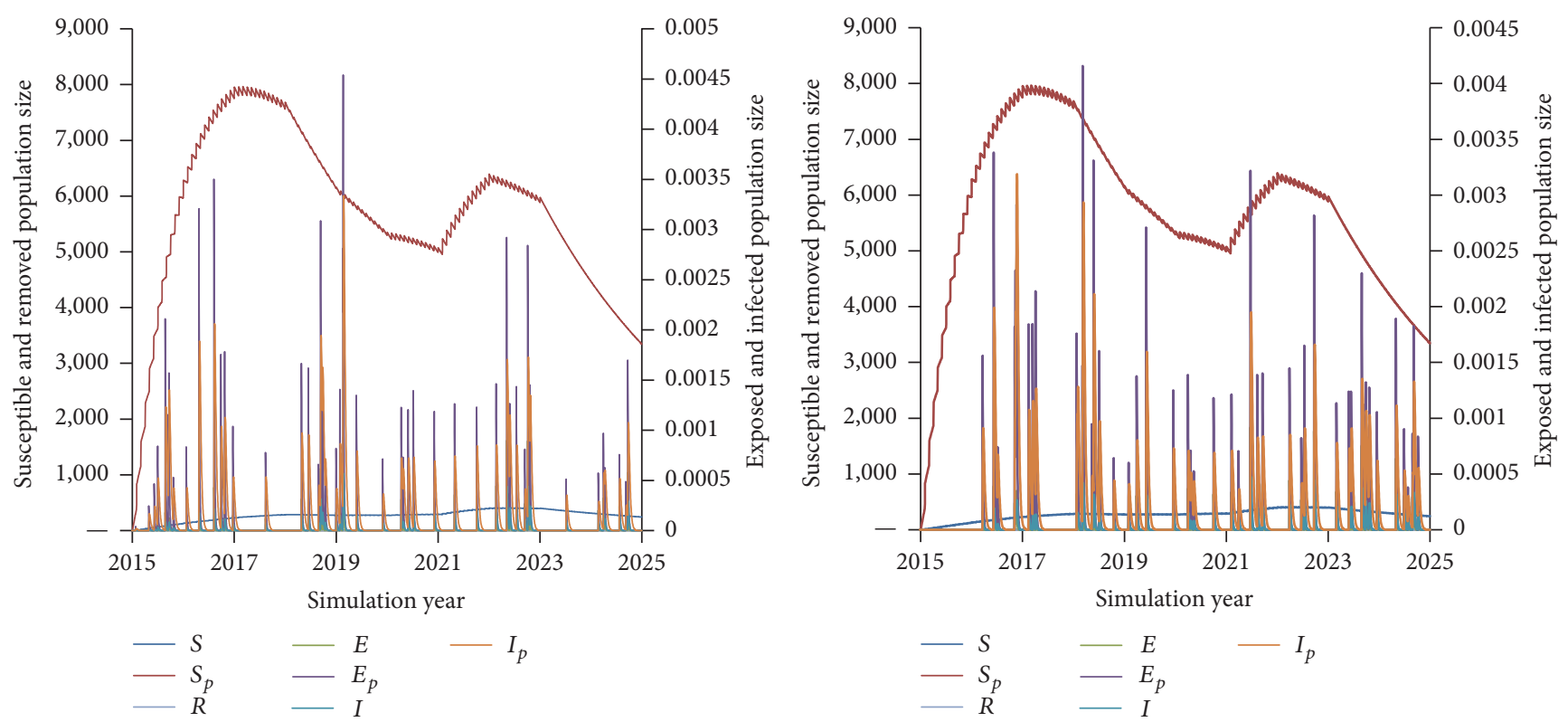

(a)

(b)

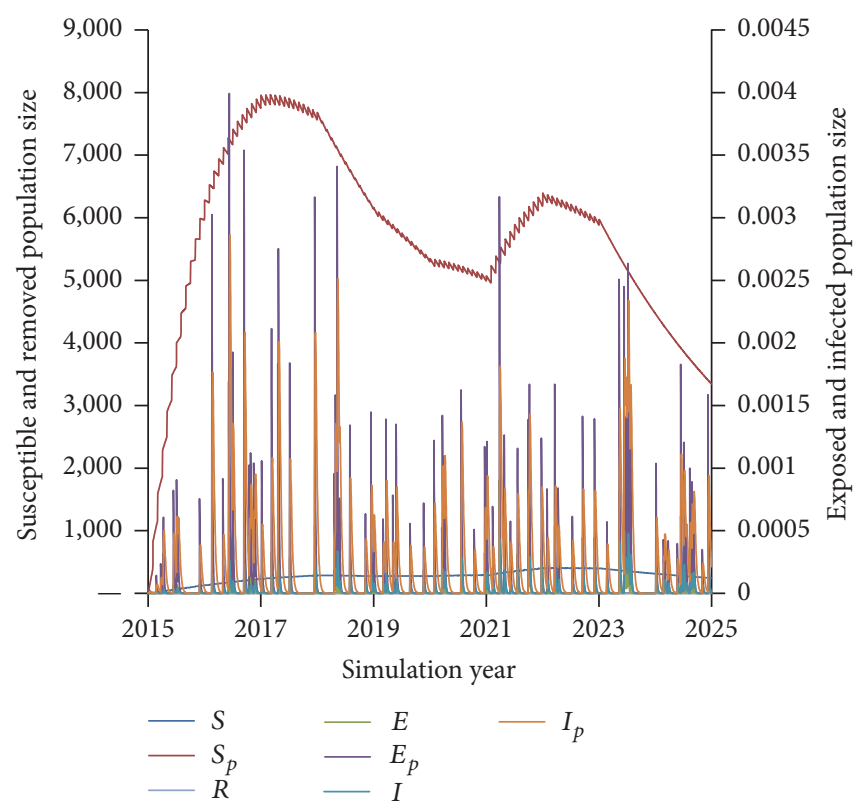

(c)

FIGURE 5: Polio disease dynamics among deployed military populations under military immunization Scenarios (a) 1 (blanket + booster + residual childhood), (b) 2 (booster + residual childhood), and (c) 3 (residual childhood).

TABLE 6: Modelled risk of new polio infections among nondeployed service members as a result of infected soldiers reintegrating upon return from deployment.

\begin{tabular}{lccccc}
\hline Scenario & $\begin{array}{c}\text { \% protected from } \\
\text { disease } \\
\text { (deployed) }\end{array}$ & $\begin{array}{c}\text { Average annual } \\
\text { incidence } \\
\text { (nondeployed) }\end{array}$ & $\begin{array}{c}\text { \% protected from } \\
\text { disease } \\
\text { (nondeployed) }\end{array}$ & $\begin{array}{c}\text { \% susceptible to } \\
\text { transmission } \\
\text { (nondeployed) }\end{array}$ & $\begin{array}{c}\text { Risk of new infections } \\
\text { (nondeployed) }\end{array}$ \\
\hline 1 & $99 \%$ & $1.2 / 100,000$ & $99 \%$ & $21 \%$ & $0.000504 / 1,000,000$ \\
2 & $99 \%$ & $1.2 / 100,000$ & $99 \% \searrow 93 \%$ & $21 \% \nearrow 26 \%$ & $0.000504 / 1,000,000 \nearrow$ \\
3 & & & & $0.000624 / 1,000,000$ \\
3 & $99 \% \succ 93 \%$ & $1.3 / 100,000$ & $99 \% \searrow 93 \%$ & $21 \% \nearrow 26 \%$ & $0.000546 / 1,000,000 \nearrow$ \\
& & & & & $0.000676 / 1,000,000$ \\
\hline
\end{tabular}




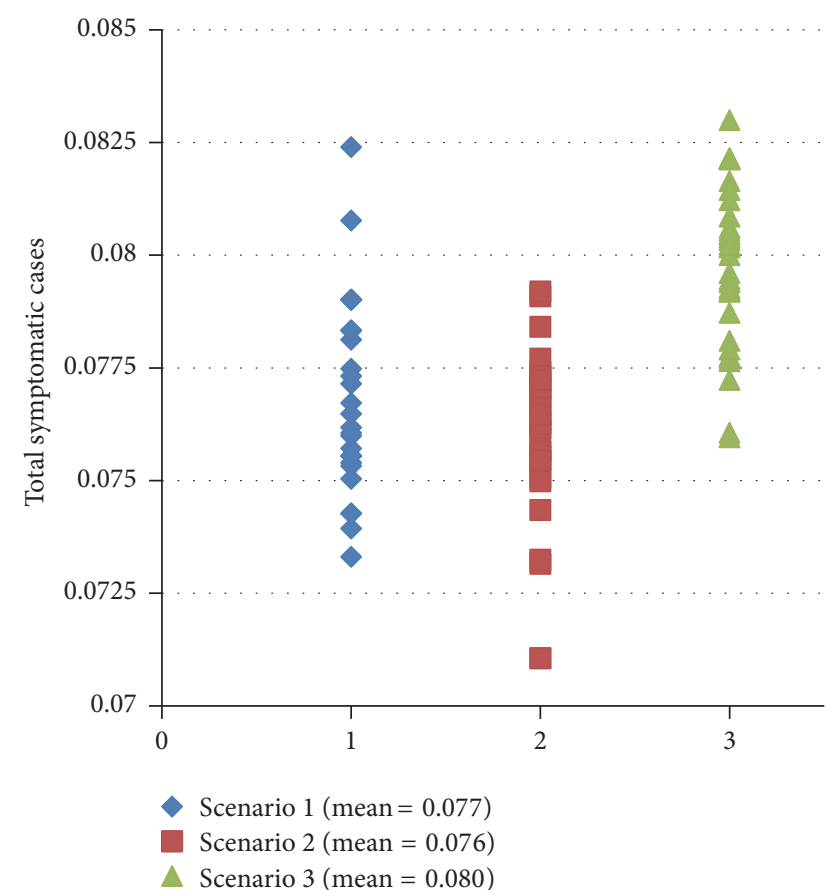

(a)

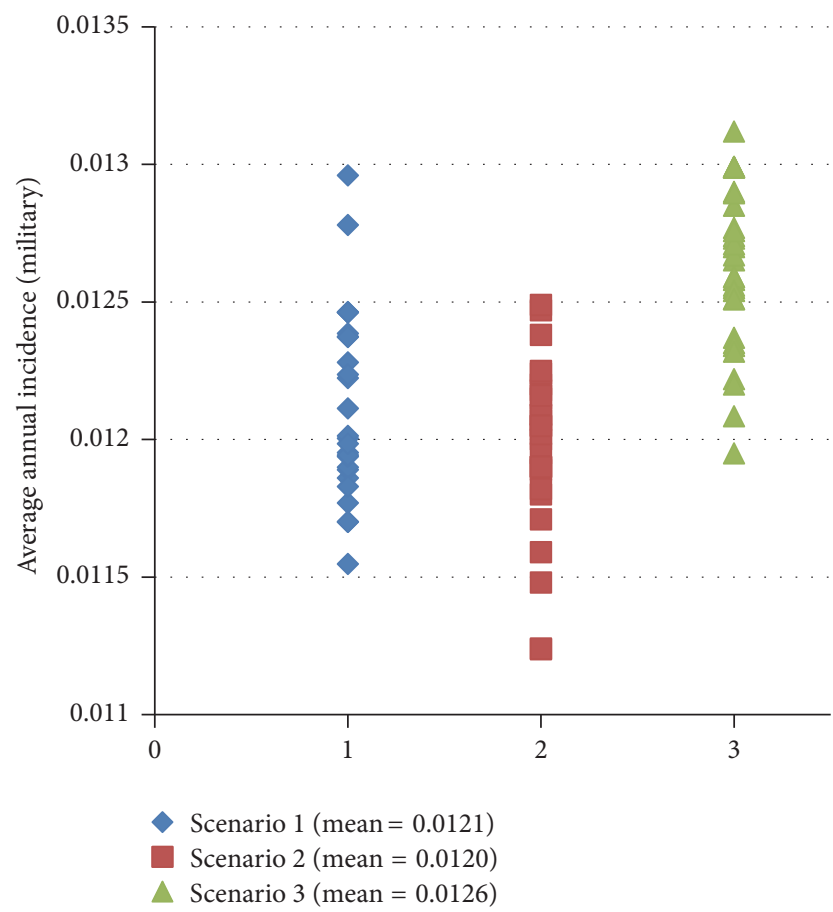

(c)

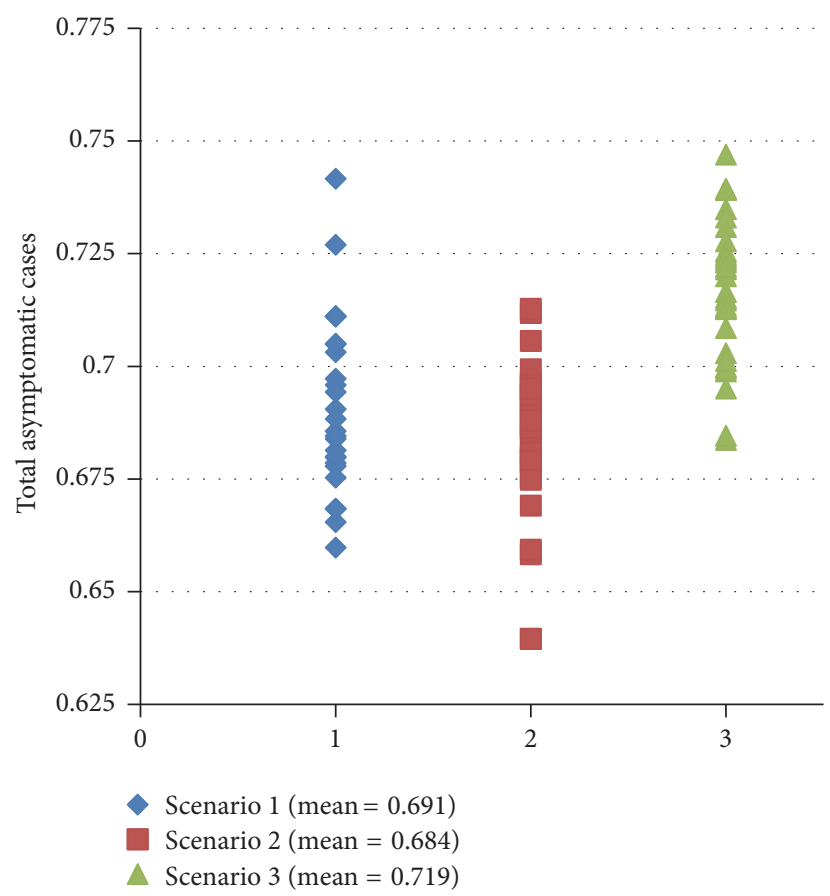

(b)

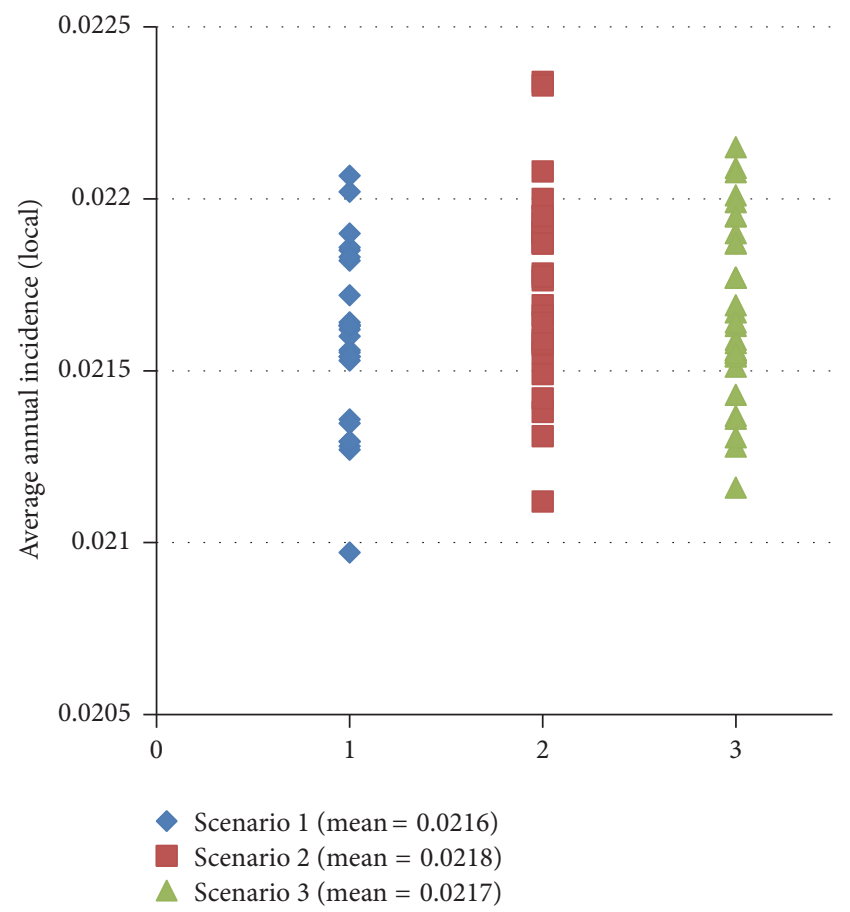

(d)

FIGURE 6: Distribution over 1500 simulations per scenario of (a) total symptomatic polio cases in deployed military populations; (b) total asymptomatic polio cases in deployed military populations; (c) average annual polio incidence in deployed military populations; and (d) average annual polio incidence in local populations under 3 military immunization scenarios.

overall risk of new infections among both deployed and nondeployed service members was extremely low, resulting from the combination of high US childhood immunization coverage rates, conferment of partial protection against polio transmission by IPV, and low disease incidence levels globally. At this range of risk, the likelihood of importation of polio cases among deployed soldiers, and subsequent spread to their nondeployed counterparts, is exceptionally small even in the absence of blanket immunization.

Given preexisting protection resulting from routine childhood vaccination, predeployment booster of service members driven by travel to polio-endemic regions is 
sufficient to prevent additional transmission among both deployed and nondeployed populations based on these results. Blanket mandatory polio vaccination of Department of Defense service members appears to be epidemiologically redundant, and dropping this routine immunization will not adversely affect troop readiness or mission objectives.

\section{Disclosure}

Kellie McMullen is a military service member (or employee of the US Government). This work was prepared as part of the official duties. Title 17, USC $\$ 105$, provides that "copyright protection under this title is not available for any work of the United States Government." Title 17, USC \$101, defines a US Government work as work prepared by a military service member or employee of the US Government as part of that person's official duties. Report no. 17-XX is supported by the Military Vaccine Agency under Work Unit no. N1415. The views expressed in this article are those of the authors and do not necessarily reflect the official policy or position of the Department of the Navy, Department of the Army, Department of the Air Force, Department of Veterans Affairs, Department of Defense, or the US Government. This work is approved for public release; distribution is unlimited.

\section{Conflicts of Interest}

The authors declare they have no conflicts of interest.

\section{Acknowledgments}

This work was funded by the Military Vaccine Agency, US Government Work (17 USC 105), and NHRC Contract no. N62645-15-F-1002. The authors wish to thank LCDR Lori N. Perry for her assistance with this contract and manuscript.

\section{References}

[1] World Health Organization, "Poliomyelitis, Fact Sheet No. 114," 2017, http://www.who.int/mediacentre/factsheets/ fs114/en/index.html.

[2] Global Polio Eradication Initiative, "Data and monitoring: Polio this week as of 2 March 2016," 2016, http://www .polioeradication.org/Dataandmonitoring/Poliothis-week.aspx.

[3] World Health Organization, "Polio vaccines and polio immunization in the pre-eradication era: WHO position paper," Weekly Epidemiological Record, vol. 85, pp. 213-228, 2010.

[4] K. M. Thompson, R. J. Duintjer Tebbens, and M. A. Pallansch, "Evaluation of response scenarios to potential polio outbreaks using mathematical models," Risk Analysis, vol. 26, no. 6, pp. 1541-1556, 2006.

[5] Global Polio Eradication Initiative, "Fact Sheet: Vaccinederived poliovirus," 2015, http://www.polioeradication.org/ Portals/0/Document/Polioandprevention/CVDPVFactSheetMarch2015.pdf.

[6] D. J. Wood, R. W. Sutter, and W. R. Dowdle, "Stopping poliovirus vaccination after eradication: Issues and challenges," Bulletin of the World Health Organization, vol. 78, no. 3, pp. 347-357, 2000.
[7] M. Eichner and K. P. Hadeler, "Deterministic models for the eradication of poliomyelitis: Vaccination with the inactivated (IPV) and attenuated (OPV) polio virus vaccine," Mathematical Biosciences, vol. 127, no. 2, pp. 149-166, 1995.

[8] World Health Organization, "Statement on the 8th IHR Emergency Committee meeting regarding the international spread of poliovirus," http://www.who.int/mediacentre/news/ statements/2016/8th-IHR-emergency-committee-polio/en/.

[9] R. J. D. Tebbens, M. A. Pallansch, O. M. Kew, V. M. Cáceres, R. W. Sutter, and K. M. Thompson, "A dynamic model of poliomyelitis outbreaks: Learning from the past to help inform the future," American Journal of Epidemiology, vol. 162, no. 4, pp. 358-372, 2005.

[10] World Health Organization, "Status updates on country planning for IPV introduction," http://www.who.int/entity/ immunization/diseases/poliomyelitis/endgame_objective2/ IPV_2016_November.pptx?ua=1.

[11] Global Polio Eradication Initiative, "This Week: Polio this week as of 12 April 2017," http://polioeradication.org/ polio-today/polio-now/this-week.

[12] H. Rahmandad, K. Hu, R. J. D. Tebbens, and K. M. Thompson, "Development of an individual-based model for polioviruses: Implications of the selection of network type and outcome metrics," Epidemiology and Infection, vol. 139, no. 6, pp. 836848,2011

[13] Centers for Disease Control and Prevention, "Vaccines and preventable diseases: polio vaccination," http://www.cdc.gov/ vaccines/vpd-vac/polio/default.htm.

[14] V. Kak, "Infections in Confined Spaces: Cruise Ships, Military Barracks, and College Dormitories," Infectious Disease Clinics of North America, vol. 21, no. 3, pp. 773-784, 2007.

[15] C. K. Murray and L. L. Horvath, "An approach to prevention of infectious diseases during military deployments," Clinical Infectious Diseases, vol. 44, no. 3, pp. 424-430, 2007.

[16] R. J. D. Tebbens, M. A. Pallansch, J.-H. Kim et al., "Oral poliovirus vaccine evolution and insights relevant to modeling the risks of circulating vaccine-derived polioviruses (cVDPVs)," Risk Analysis, vol. 33, no. 4, pp. 680-702, 2013.

[17] M. R. Behrend, H. Hu, K. R. Nigmatulina, and P. Eckhoff, "A quantitative survey of the literature on poliovirus infection and immunity," International Journal of Infectious Diseases, vol. 18, pp. 4-13, 2014.

[18] J.-H. Kim and S.-H. Rho, "Transmission dynamics of oral polio vaccine viruses and vaccine-derived polioviruses on networks," Journal of Theoretical Biology, vol. 364, pp. 266-274, 2015.

[19] K. M. Thompson, M. A. Pallansch, R. J. D. Tebbens, S. G. Wassilak, and S. L. Cochi, "Modeling population immunity to support efforts to end the transmission of live polioviruses," Risk Analysis, vol. 33, no. 4, pp. 647-663, 2013.

[20] D. A. Kalkowska, R. J. Duintjer Tebbens, I. Grotto et al., "Modeling options to manage type 1 wild poliovirus imported into Israel in 2013," Journal of Infectious Diseases, vol. 211, no. 11, pp. 1800-1812, 2015.

[21] D. A. Kalkowska, R. J. D. Tebbens, M. A. Pallansch, S. L. Cochi, S. G. Wassilak, and K. M. Thompson, "Modeling undetected live poliovirus circulation after apparent interruption of transmission: Implications for surveillance and vaccination," $B M C$ Infectious Diseases, vol. 15, no. 1, 66 pages, 2015.

[22] J. Koopman, C. Henry, J. Park, M. Eisenberg, E. Ionides, and J. Eisenberg, "Dynamics affecting the risk of silent circulation when oral polio vaccination is stopped," Epidemics, 2017. 
[23] C. Burgess, A. Peace, R. Everett, B. Allegri, and P. Garman, "Computational modeling of interventions and protective thresholds to prevent disease transmission in deploying populations," Computational and Mathematical Methods in Medicine, Article ID 785752, Art. ID 785752, 17 pages, 2014.

[24] "United Nations, Department of Economic and Social Affairs, Population Division, World Population Prospects, the 2015 Revision," http://esa.un.org/unpd/wpp/DVD.

[25] World Health Organization, "WHO/UNICEF estimates of national routine immunization coverage, 2013 revision," http:// www.who.int/immunization/monitoring_surveillance/data/en/.

[26] R. J. Duintjer Tebbens, M. A. Pallansch, D. A. Kalkowska, S. G. F. Wassilak, S. L. Cochi, and K. M. Thompson, "Characterizing poliovirus transmission and evolution: Insights from modeling experiences with wild and vaccine-related polioviruses," Risk Analysis, vol. 33, no. 4, pp. 703-749, 2013.

[27] I. Blake and K. O’Reilly, "Approaches to modeling, parameter estimation, and policy guidance during the endgame," in Proceedings of the Workshop on Analyzing the Polio Eradication Endgame, Seattle, WA, USA, July 2015.

[28] Centers for Disease Control and Prevention, Epidemiology and Prevention of Vaccine-Preventable Diseases, Public Health Foundation, Washington D.C, Wash, USA, 13th edition, 2015.

[29] N. Nathanson and O. M. Kew, "From emergence to eradication: the epidemiology of poliomyelitis deconstructed," American Journal of Epidemiology, vol. 172, no. 11, pp. 1213-1229, 2010.

[30] H. E. Jenkins, R. B. Aylward, A. Gasasira et al., "Implications of a circulating vaccine-derived poliovirus in Nigeria," New England Journal of Medicine, vol. 362, pp. 2360-2369, 2010.

[31] D. R. Prevots, M. L. Ciofi Degli Atti, A. Sallabanda et al., "Outbreak of paralytic poliomyelitis in Albania, 1996: high attack rate among adults and apparent interruption of transmission following nationwide mass vaccination," Clinical Infectious Diseases, vol. 26, no. 2, pp. 419-425, 1998.

[32] J. R. Paul and D. M. Horstmann, "A survey of poliomyelitis virus antibodies in French Morocco," The American journal of tropical medicine and hygiene, vol. 4, no. 3, pp. 512-524, 1955.

[33] H. Jafari, J. M. Deshpande, R. W. Sutter et al., "Efficacy of inactivated poliovirus vaccine in India," Science, vol. 345, no. 6199, pp. 922-925, 2014.

[34] World Health Organization, "Reported cases of selected vaccine preventable diseases (VPDs)," http://www.who.int/entity/ immunization/monitoring_surveillance/data/incidence_series .xls?ua $=1$.

[35] K. Lapinleimu and M. Stenvik, "Experiences with polio vaccination and herd immunity in Finland," Developments in Biological Standardization, vol. 47, pp. 241-246, 1981.

[36] Anonymous, "2012 Demographics Profile of the Military Community. Office of the Deputy Assistant Secretary of Defense (Military Community and Family Policy)," http:// download.militaryonesource.mil/12038/MOS/Reports/2012 Demographics_Report.pdf.

[37] Centers for Disease Control and Prevention, "Vaccination coverage among children in kindergarten - United States, 201213 school year," Morbidity and Mortality Weekly Report, vol. 62, no. 30, pp. 607-612, 2013.

[38] Headquarters Departments of the Army, the Navy, the Air Force, and the Coast Guard, Medical Services, "Immunizations and chemoprophylaxis for the prevention of infectious disease," 2013, http://www.apd.army.mil/jw2/xmldemo/ r40_562/main.asp.
[39] Congress of the United States Congressional Budget Office, "Recruiting, retention, and future levels of military personnel," October 2006, https://www.cbo.gov/sites/default/files/ cbofiles/ftpdocs/76xx/doc7626/10-05-recruiting.pdf. 


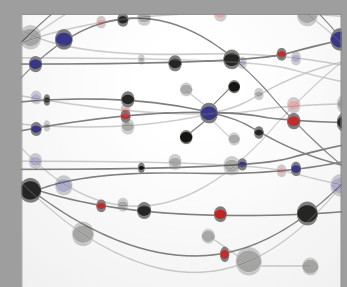

The Scientific World Journal
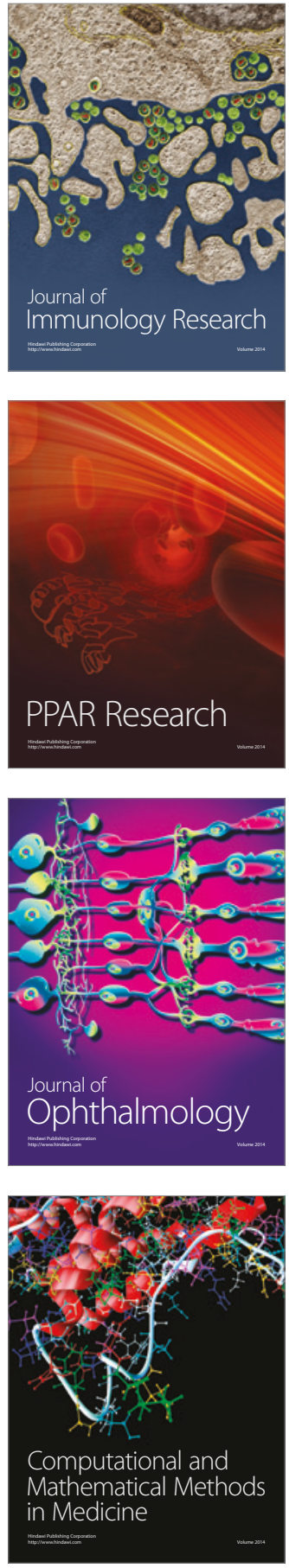

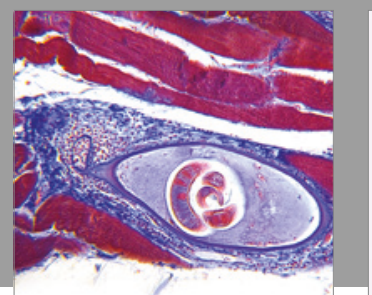

Gastroenterology Research and Practice
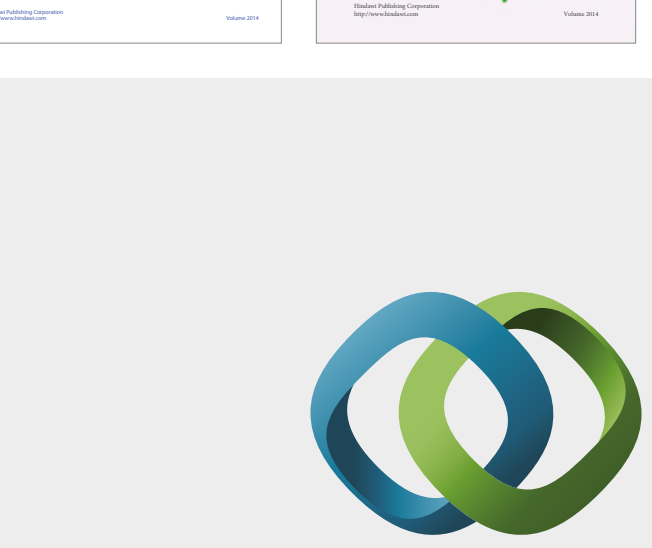

\section{Hindawi}

Submit your manuscripts at

https://www.hindawi.com
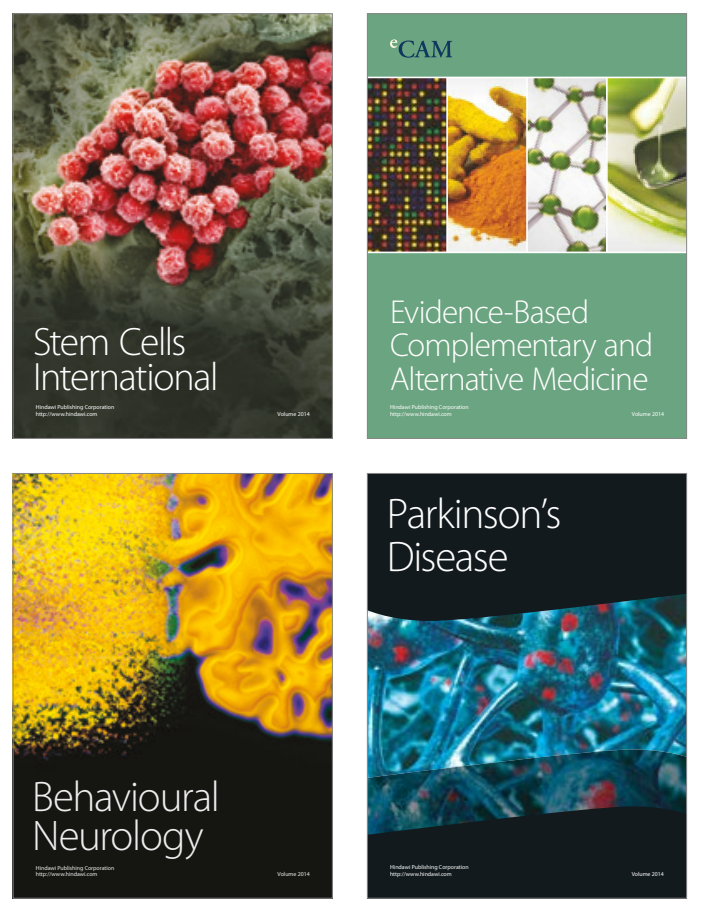
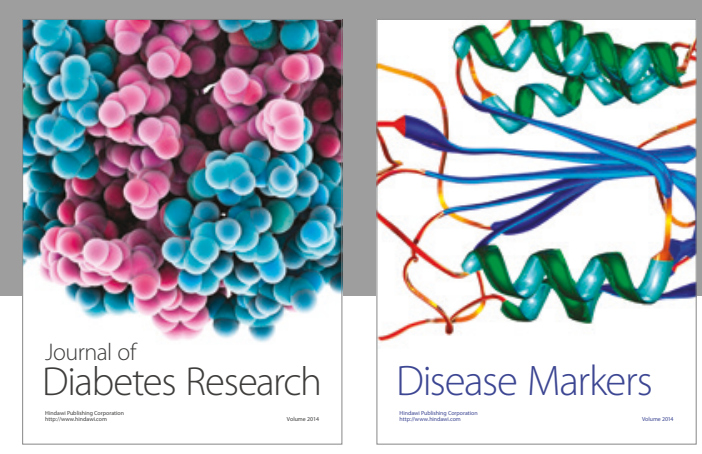

Disease Markers
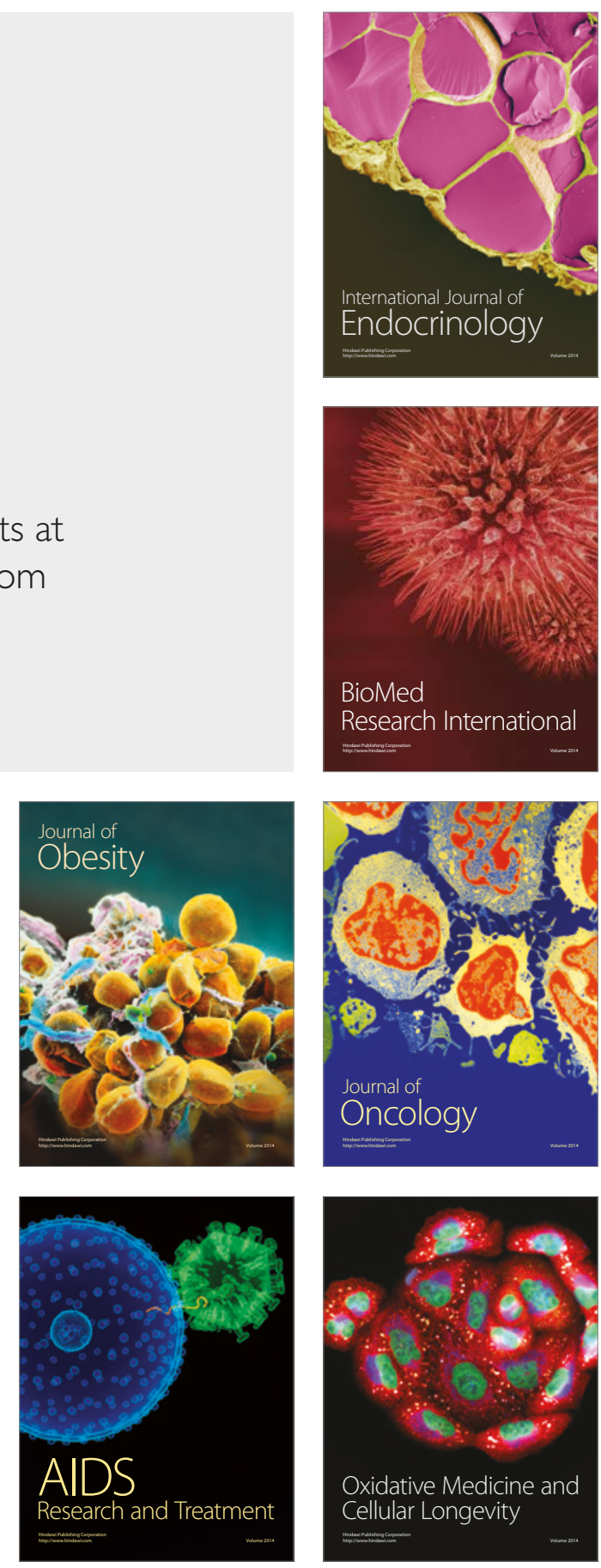